\title{
Structure Optimization and Numerical Simulation of Nozzle for High Pressure Water Jetting
}

\author{
Shuce Zhang, ${ }^{1}$ Xueheng Tao, ${ }^{1,2}$ Jinshi Lu, ${ }^{1,2}$ Xuejun Wang, ${ }^{1,2}$ and Zhenhua Zeng ${ }^{3}$ \\ ${ }^{1}$ School of Mechanical Engineering and Automation, Dalian Polytechnic University, Dalian 116034, China \\ ${ }^{2}$ National Engineering Research Center of Seafood, Dalian 116034, China \\ ${ }^{3}$ Dalian Modern Auxiliary Machines Development and Manufacturing Co., Ltd., Dalian 116600, China \\ Correspondence should be addressed to Xueheng Tao; xhtao@dlpu.edu.cn
}

Received 4 November 2014; Revised 17 January 2015; Accepted 27 January 2015

Academic Editor: Marjan Bahraminasab

Copyright (C) 2015 Shuce Zhang et al. This is an open access article distributed under the Creative Commons Attribution License, which permits unrestricted use, distribution, and reproduction in any medium, provided the original work is properly cited.

\begin{abstract}
Three kinds of nozzles normally used in industrial production are numerically simulated, and the structure of nozzle with the best jetting performance out of the three nozzles is optimized. The $R 90$ nozzle displays the most optimal jetting properties, including the smooth transition of the nozzle's inner surface. Simulation results of all sample nozzles in this study show that the helix nozzle ultimately displays the best jetting performance. Jetting velocity magnitude along $Y$ and $Z$ coordinates is not symmetrical for the helix nozzle. Compared to simply changing the jetting angle, revolving the jet issued from the helix nozzle creates a grinding wheel on the cleaning surface, which makes not only an impact effect but also a shearing action on the cleaning object. This particular shearing action improves the cleaning process overall and forms a wider, effective cleaning range, thus obtaining a broader jet width.
\end{abstract}

\section{Introduction}

In the process of material surface cleaning by high pressure water jet, the enhancement of cleaning effectiveness and reduction of energy consumption have been significant areas of research for quite some time. Nurick (1976) studied the cavitation characteristics of sharp-edged orifices including circular orifices and rectangular orifices and their effect on spray mixing [1]. Hashish and du Plessis (1978) developed a theory based on a control volume analysis, which presented a basic method for building cutting equations to evaluate hydrodynamic forces [2]. Soyama et al. (1996) focused on peculiar phenomena occurring around high speed submerged water jets, clarified the effectiveness of the nozzle structure and injection pressure therein, and then discussed the observed characteristics and configuration of the water jet [3]. A clear relationship between parameters (standoff distance and installation angle) and cleaning results was drawn by Gao and Chen (2006) [4].

Estimation in nozzle cleaning performance is of paramount importance in high axial velocity and radial velocity. We hold the opinion based on experiment and analysis that different nozzle configurations determined different axial velocities. Considering shearing action will function in cleaning process, we have made a contribution to increasing axial and radial velocity by optimizing nozzle configuration. This study, which focuses on nozzle configuration, posits a new nozzle and verifies its highly effective jet velocity by numerical simulation. Three kinds of nozzles commonly used in industrial production, the Cylindrical Contracting (CC) nozzle, Taper Contracting (TC) nozzle, and Stepped nozzle, are simulated by FLUENT to obtain exact jet velocity comparison data. The most applicable of these is then sequentially optimized by simulating tentative structure design models. For the sequential optimization, a 3D model is used to simulate the helix nozzle outlet structure. Comparison results from the simulation of different nozzle inner surface configurations indicate the most favorable, best performing nozzle structure.

\section{Materials and Methods}

2.1. Preliminary Optimization of Nozzle Structure. The nozzle structure determines the water jet velocity, which also 


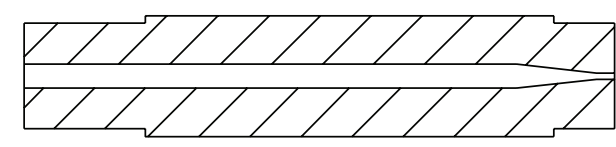

(a) CC nozzle

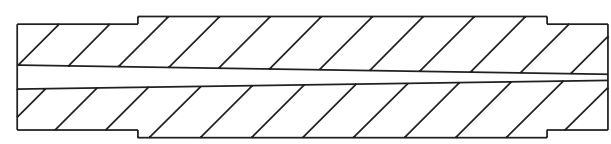

(b) TC nozzle

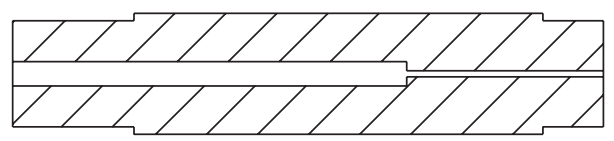

(c) Stepped nozzle

FIgURE 1: Nozzle structures.

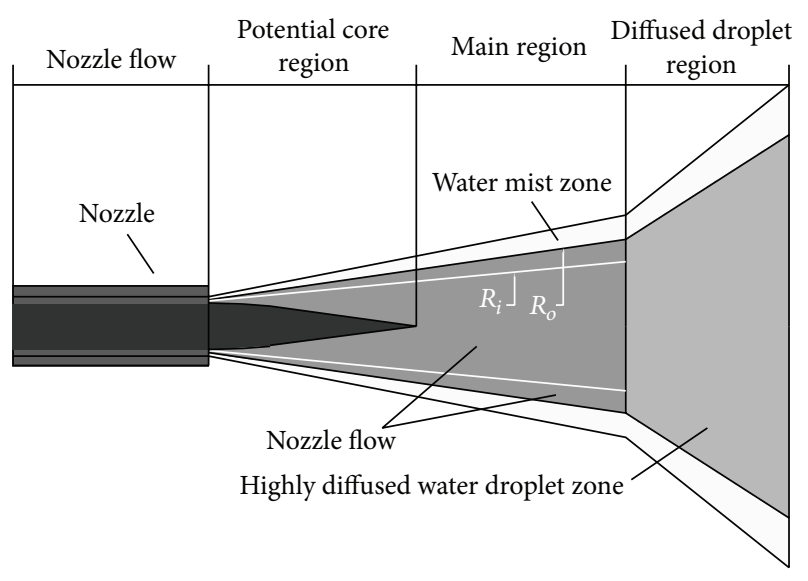

FIGURE 2: Jet structure based on theory and observation [6].

determines the cleaning process. The Cylindrical Contracting (CC) nozzle, Taper Contracting (TC) nozzle, and Stepped nozzle are those generally used in the industry currently. Figure 1 details their respective structures. The type of water jet that issues from all these different orifice nozzles has similar jet structures in air. Yanaida and Ohashi first detailed a geometrical description of the water jet in 1980 [5].

Over the past 40 years, a recognizable characteristic pattern has emerged. Jet structure, based on theory and observation, is shown in Figure 2 [6]. The jet velocity is equally distributed at the nozzle but produces a considerable velocity difference as soon as it leaves the nozzle, compared to the boundary layer formed by the environment medium. The process of exchanging momentum and mass of jet medium and environment medium is the same as the process of diffusing jet fluid.

2.1.1. Potential Core Region. Flow within this region displays irrotational motion. Velocity gradients, either horizontal or vertical, do not exist inside of the potential core.

2.1.2. Main Region. Within the segment, jet axial velocity and dynamic pressure reduce gradually, and turbulence characteristics are strong. Discrepancy between the potential core region and this turbulent mixing region, which is shaped by the jet medium and environment medium, fails to achieve statistical significance.

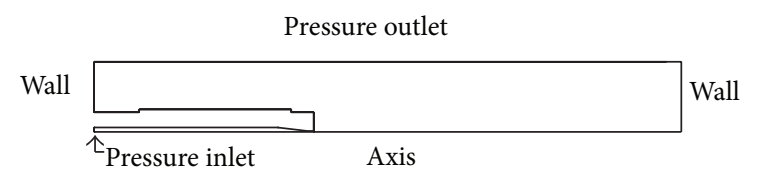

FIgURE 3: Computational area and boundary conditions of CC nozzle in GAMBIT.

2.1.3. Diffused Droplet Region. Jet medium and environment medium are thoroughly mixed. The jet mainly loses cohesion. Axial velocity and dynamic pressure are relatively low.

The jet in the potential core region is applied to cutting, and jetting in the diffused droplet region is applied to dustlaying and aspirating, whereas the jet in the main region, the focus of this study, is applied to cleaning and surface finish. Nozzles shown in Figure 1 are widely used in engineering projects; however, higher velocity, higher dynamic pressure, lower pump pressure, and lower cost are necessary considerations. The optimization of nozzle structure offers a simple solution to these. The most suitable nozzle is selected for the study from the three kinds of nozzles listed above.

FLUENT was used as the simulation tool. Two-dimensional simulation of the jet formation of the CC nozzle, TC nozzle, and Stepped nozzle was performed first. The computational area and boundary conditions were as shown in Figure 3. The structured grid system of the nozzles was created in GAMBIT. The inlet boundary condition was a pressure inlet, and the outlet boundary condition was a pressure outlet. Water-liquid, the subject of flow in axisymmetric space, was the uncompressed ideal fluid and followed a steady flow pattern.

Figure 4 shows the main nozzle structural parameters. According to the theoretical analysis and experimental data, geometric parameters of the CC nozzle are defined as $\alpha=$ $13^{\circ}, l / d=3$, and $D / d=4$. Boundary conditions were considered the inlet pressure $=100 \mathrm{MPa}$ and outlet pressure of normal atmospheric pressure. The fluent fluid material was water-liquid, and the standard $k-\varepsilon$ model is appropriate in our case. Nozzle outlet diameter was $0.8 \mathrm{~mm}$, inlet diameter was $3.2 \mathrm{~mm}$, and computational area size was $288 \times 25 \mathrm{~mm}^{2}$ to ensure that the water jet was completely divergent. The numerical methodology expressed by Tu et al. (2007) was adopted in our flow model [7]. The flow velocity at the inlet 


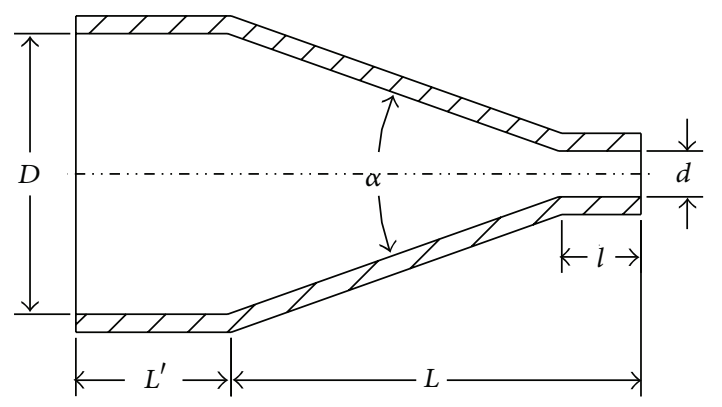

FIGURE 4: Main nozzle structural parameters.

and outlet could be preliminarily roughly calculated based on the Bernoulli equation:

$$
\frac{p_{1}}{\gamma}+\frac{v_{1}^{2}}{2 g}=\frac{p_{2}}{\gamma}+\frac{v_{2}^{2}}{2 g},
$$

where $p_{1}, p_{2}$ are pressures at inlet and outlet cross sections along the horizontal pipeline of the nozzle, $v_{1}, v_{2}$ are flow velocities at the two cross sections, and $\gamma$ denotes flow specific weight.

The equation for counting volume flux in the pipeline is then deduced:

$$
q=1.2 q_{0}
$$

According to the definition $q=v A$, (1) is then written as follows:

$$
\left(\frac{1}{2 g A_{1}^{2}}-\frac{1}{2 g A_{2}^{2}}\right) q_{0}^{2}=\frac{1}{\gamma}\left(p_{2}-p_{1}\right) \text {. }
$$

The term $q_{0}$ is a theoretical value of volume flux. Considering friction loss, more accurate volume flux $q$ is obtained from $q_{0}$ multiplying by a friction coefficient (set to 1.2). $A_{1}, A_{2}$ are areas of the two cross sections. Certain concrete values must be used to analyze our flow model, among which $A_{1}$ is inlet cross section area and $A_{2}$ is outlet cross section area, defined as follows: $A_{1}=\pi R^{2}, A_{2}=\pi r^{2}$.

By substituting (3) into (2) and adding the actual values to these equations, we obtain

$$
\begin{gathered}
v_{1}=28 \mathrm{~m} / \mathrm{s} ; \\
v_{2}=447.85 \mathrm{~m} / \mathrm{s} .
\end{gathered}
$$

Theoretical values $v_{1}$ and $v_{2}$, slightly different compared to the values, are used for quantitative analysis in distinguishing flow type. One important use of the Reynolds number (Re) obtained by $v_{1}$, regarded an essential nondimensional parameter that describes the flow characteristics, is to indicate whether the flow is turbulent or laminar. A channel flow will become turbulent if the critical Reynolds number is above 1400. Re is defined as the ratio of the inertia force over the friction force:

$$
\operatorname{Re}=\frac{\text { Inertia Force }}{\text { Friction Force }}=\frac{\rho V_{1} D}{\mu} .
$$

It is observed that the equation encapsulates three variables, density $(\rho)$, dynamic viscosity $(\mu)$, and inlet velocity $\left(v_{1}\right) \cdot D(=$ $2 R)$ is the diameter of the nozzle inlet. Combining (1) and (3) into (5), we obtain

$$
\operatorname{Re}=9.0 \times 10^{4} \gg 1400 .
$$

Thus, we confirm that the flow model is turbulent. Our case is a constant property fluid flow, indicating that flow density is constant and body force in the equation can be neglected. The momentum equations employed are given as follows [8]:

$$
\begin{aligned}
& \frac{\partial\left(\alpha_{w} \rho_{w}\right)}{\partial t}+\nabla \cdot\left(\alpha_{w} \rho_{w} \vec{v}_{w}\right) \\
& =\sum_{i=w, a}\left(\dot{m}_{a \rightarrow w}-\dot{m}_{w \rightarrow a}\right)+S_{w} \\
& \frac{\partial\left(\alpha_{w} \rho_{w} \vec{v}_{w}\right)}{\partial t}+\nabla \cdot\left(\alpha_{w} \rho_{w} \vec{v}_{w} \vec{v}_{w}\right) \\
& =\sum_{i=w, a}\left\{K_{w a}\left(\vec{v}_{w}-\vec{v}_{a}\right)+\dot{m}_{a \rightarrow w} \vec{v}_{a \rightarrow w}-\dot{m}_{w \rightarrow a} \vec{v}_{w \rightarrow a}\right\} \\
& \quad-\alpha_{w} \nabla p+\nabla \cdot \overline{\bar{\tau}}_{w}+\alpha_{w} \rho_{w} \vec{g}+\vec{F}_{w},
\end{aligned}
$$

where $\alpha_{w}$ is the volume fraction; $\rho_{w}$ is the density of water; and $v_{w}$ is the axial velocity of water droplets. The term $\dot{m}_{w \rightarrow a}$ is the mass transfer from $w$ (water) phase. In order to simulate the air entrainment process, we set $\dot{m}_{w \rightarrow a}$ and $S_{w}$ as zero and

$$
\dot{m}_{w \rightarrow a}=\nabla \cdot(\dot{M}, 0),
$$

where $\dot{M}$ is known from the semiempirical mass model of Guha et al. [9]:

$$
\begin{gathered}
\dot{M}(x, y)=\frac{5.62 \times \rho_{w} \times \alpha_{w 0} \times V_{w 0} \times r^{2}}{r_{x}^{2}} \\
\cdot\left\{1-\left(\frac{y}{r_{x}}\right)^{1.5}\right\}^{3},
\end{gathered}
$$

where $\alpha_{w 0}$ and $V_{w 0}$ are the volume fraction and axial velocity of water droplets at the nozzle exit, respectively, and $r$ is the nozzle radius. The variable $r_{x}$ is defined as

$$
\begin{gathered}
r_{x}=R_{i} \quad \text { if } y \leq R_{i}, \\
r_{x}=R_{0} \quad \text { if } R_{i}<y \leq R_{0},
\end{gathered}
$$

where $R_{i}$ is the radial width of the continuous flow region and is given by

$$
R_{i}=k_{1} \sqrt{x r}+r
$$

Outside of this region is the droplet flow region, the radial width of which varies as follows:

$$
R_{0}=C x+r .
$$


$k_{1}$ and $C$ are spread coefficients. The value of $C$ is usually in between 0.02 and $0.06 . k_{1}$ is estimated as follows:

$$
k_{1} \sqrt{r}=\text { const } \times C \text {, }
$$

where const is usually $O\left(10^{-1}\right)$. Schematic description of $R_{i}$ and $R_{0}$ has been provided in Figure 2 .

The standard $k-\varepsilon$ model equations, expressed in nonconservation form, cause an increase in demand. The transport equations are as follows:

$$
\begin{gathered}
\frac{\partial\left(\rho_{m} k\right)}{\partial t}+\nabla \cdot\left(\frac{\mu_{t, m}}{\sigma_{k}} \nabla k\right)+G_{k, m}-\rho_{m} \varepsilon \\
\frac{\partial\left(\rho_{m} \varepsilon\right)}{\partial t}+\nabla \cdot\left(\rho_{m} \vec{v}_{m}\right)=\nabla \cdot\left(\frac{\mu_{t, m}}{\sigma_{k}} \nabla \varepsilon\right) \\
+\frac{\varepsilon}{k}\left(c_{\varepsilon 1} G_{k, m}-c_{\varepsilon 2} \rho_{m} \varepsilon\right),
\end{gathered}
$$

where $\rho_{m}$ is the mixture density and $\vec{v}_{m}$ is the mixture velocity. The turbulent viscosity $\left(\mu_{t, m}\right)$ and the production of turbulent kinetic energy $\left(G_{k, m}\right)$ are calculated as follows:

$$
\begin{gathered}
\mu_{t, m}=\rho_{m} c_{\mu} \frac{k^{2}}{\varepsilon}, \\
G_{k, m}=\mu_{t, m}\left(\nabla \vec{v}_{m}+\left(\nabla \vec{v}_{m}\right)^{T}\right) .
\end{gathered}
$$

The adjustable constants $c_{\mu}, \sigma_{k}, \sigma_{\varepsilon}, c_{\varepsilon 1}$, and $c_{\varepsilon 2}$ are obtained by sufficient data statistics (Launder and Spalding, 1974) [10]:

$$
\begin{gathered}
c_{\mu}=0.09, \quad c_{\varepsilon 1}=1.44, \quad c_{\varepsilon 2}=1.92, \\
\sigma_{k}=1.0, \quad \sigma_{\varepsilon}=1.3 .
\end{gathered}
$$

2.2. Optimization Structure of Helix Nozzle. To better understand the principles at work in this study, let us consider the firing of a bullet. Propelled by gunpowder, the bullet accelerates and fires through air in a rotational motion. The pressure of powder gases expels the bullet, and it continues along a predictable trajectory. As shown in Figure 8, by observing the inside of the gun barrel, the bullet expelled does not move in a straight line but a spiral at the angles which correspond to the centerline of the barrel. It functions similarly to a top-heavy gyroscope, in the fact that it requires a rapid spinning motion to continue its trajectory. As it flies off the barrel, the bullet must overcome air resistance. A tight spiral trajectory ensures high velocity and straight, forward shooting direction.

If we think of the flow element as a bullet, as shown in Figure 5, we can effectively structure the nozzle as a gun barrel in order to simulate rotational movement. The formation of a spiral not only guides the flow, but also speeds the flow and keeps it steady. As shown in Figure 6, for precise simulation of the helix nozzle, the $2 \mathrm{D}$ model must be transformed to $3 \mathrm{D}$, as $2 \mathrm{D}$ cannot properly represent the complexity of a spiral. The $3 \mathrm{D}$ is built while keeping nozzle geometry parameters and simulating boundary conditions invariable (Table 1). A structured grid system is created in the $3 \mathrm{D}$ model, distinguished from $2 \mathrm{D}$ model by two independent mesh bodies (the nozzle and computational domain) which are connected by an interface.
TABLE 1: Values of nozzle structure parameters and boundary conditions.

\begin{tabular}{lccc}
\hline Parameter & Value & Unit & Note \\
\hline$p_{1}$ & $1.0 \times 10^{8}$ & $\mathrm{~Pa}$ & \\
$p_{2}$ & 101325 & $\mathrm{~Pa}$ & \\
$\gamma$ & 9.81 & $\mathrm{kN} / \mathrm{m}^{3}$ & Water \\
$R$ & 1.6 & $\mathrm{~mm}$ & \\
$r$ & 0.4 & $\mathrm{~mm}$ & \\
\hline
\end{tabular}

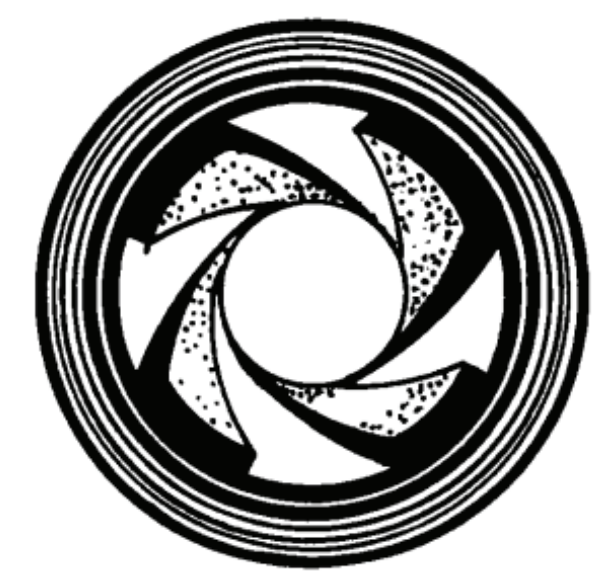

FIGURE 5: Gun barrel line structure.

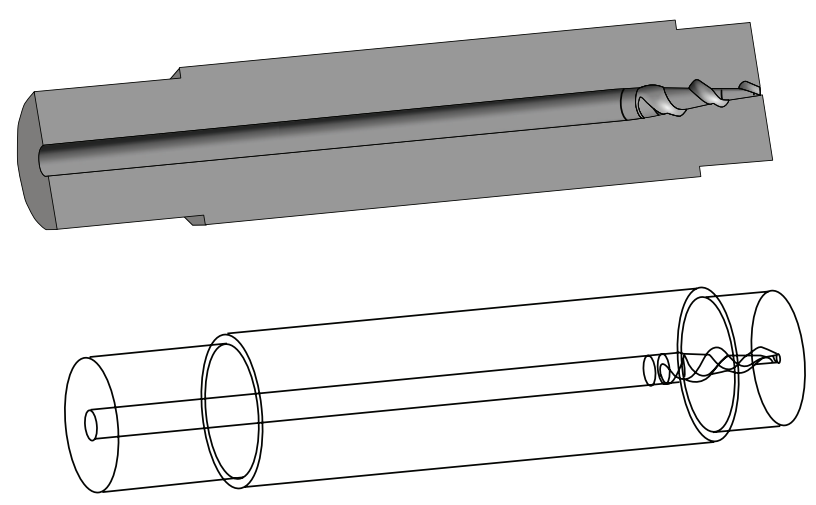

FIGURE 6: 3D structure of helix nozzle.

\section{Results and Discussion}

Nozzle structure design models with better performance were chosen to make further optimization. Table 2 shows optimization procedures of nozzle structure design models. To compare the effect of optimization design models, average axial and radial velocities of structure models in target distance range of $X=0.08 \sim 0.09 \mathrm{~m}$ are compared in Table 3 .

3.1. Preliminary Optimization. Compared to the Stepped nozzle in Figure 7, all CC nozzles and TC nozzles demonstrate better jetting performance in the range of effective target distance. Despite the fact that the highest velocity TC nozzle performed at the outlet, its velocity characteristic in the main region is similar to the CC nozzle. Evolved 
TABLE 2: Optimization procedures of nozzle structure design models.

\begin{tabular}{lcc}
\hline Optimizing solution & Object nozzles & Result \\
\hline $\begin{array}{l}\text { (1) Comparing } \\
\text { simulation }\end{array}$ & Stepped, CC, TC & CC \\
$\begin{array}{l}\text { (2) Smooth transition } \\
\text { of the inner surface }\end{array}$ & CC, $R 30, R 60, R 90$, & $R 90$ \\
$\begin{array}{l}\text { (3) Formation of a } \\
\text { spiral on the inner } \\
\text { surface }\end{array}$ & $R 120, R 150$ & \\
\hline
\end{tabular}

TABLE 3: Average axial and radial velocities of structure models in target distance range of $X=0.08 \sim 0.09 \mathrm{~m}$.

\begin{tabular}{lcc}
\hline Structure model & $\begin{array}{c}\text { Average axial velocity } \\
(\mathrm{m} / \mathrm{s})\end{array}$ & $\begin{array}{c}\text { Average radial } \\
\text { velocity }(\mathrm{m} / \mathrm{s})\end{array}$ \\
\hline Stepped nozzle & 99.8 & 3.7 \\
CC nozzle & 148.8 & 4.9 \\
TC nozzle & 151.7 & 5.1 \\
R30 nozzle & 231.6 & 7.6 \\
R60 nozzle & 134.0 & 4.6 \\
R90 nozzle & 236.1 & 7.9 \\
R120 nozzle & 139.4 & 4.9 \\
R150 nozzle & 134.7 & 4.7 \\
Helix nozzle & 205.7 & 118.8 \\
\hline
\end{tabular}

from the TC nozzle, the CC nozzle is a solid flow nozzle whose jetting behavior displays great concentrated force and standoff distance, where its shape proves beneficial in high precision micromachining. To this effect, the CC nozzle was chosen as the study object in this paper. Its configuration optimization design is processed in two stages.

As is commonly known, a sharp transition from the convergent section and the two cylindrical sections in the CC nozzle body would increase local resistance loss. As a solution, smooth transition of the inner surface can ensure fluid streamlining and form jetting that includes a high velocity coherent core.

Figure 8 shows the preliminary optimization structures, in which (a) is CC nozzle and (b)-(f) are optimized nozzles with different radii of circular arcs. These nozzles were then simulated in order to select the one with the best jetting performance. According to the different nozzle structures first attempted, these nozzles were successively simulated. Velocity magnitude was chosen for simulation, as a series of parameters such as velocity magnitude, axial velocity, dynamic pressure, and shooting force, all of which have positive correlation, are able to effectively measure jetting performance.

As shown in Figure 9, (b) is a local display of (a) at the range of $0.078 \mathrm{~m}$ to $0.10 \mathrm{~m}$ in the $X$ direction. Compared with the CC nozzle, the jetting velocity magnitude of the $R 90$ nozzle is higher than the others if the target distance was set in the range of $X=0.08 \sim 0.09 \mathrm{~m}$. A gripping tool or manipulator installed in the high pressure water jet

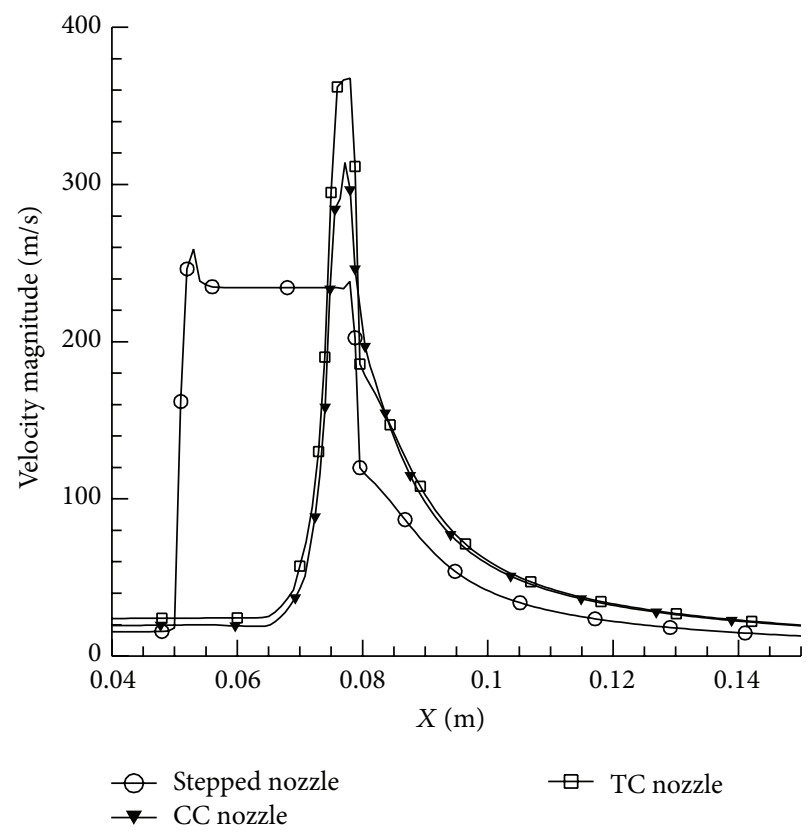

FIGURE 7: Three different nozzles structure comparison from simulation of jet velocity magnitude.

equipment enables the nozzle to reach any range of target distance; therefore, the $R 90$ nozzle is the proposed model.

3.2. Optimization of Helix Nozzle. Observing the velocity vectors as shown in Figure 10, flow velocity magnitude apparently increases along the spiral line, and horizontal velocity almost matches the axial velocity of the same cross section. Figure 11 shows that jetting velocity magnitude along $Y$ and $Z$ coordinates is not symmetrical, indicating that the high speed jet performs a shearing action on the cleaning object. In the high pressure water jet cleaning process, the jet generally loses some kinetic energy while vertically shooting at the cleaning surface. However, the jet issuing from a helix nozzle actually avoids this problem. Compared to simply changing the jetting angle, a revolving jet acts like a grinding wheel on the cleaning surface, displaying not only an impact effect but also a shearing action on the cleaning object.

In order to compare the velocity magnitude of the helix nozzle with the $R 90$ nozzle, high pressure water jetting simulation data was loaded in MATLAB, and Lagrange Interpolation Method was used to fit the velocity magnitude curves. As shown in Table 3, $R 90$ nozzle performs the highest average axial velocity but very low radial velocity; helix nozzle performs both comparatively high average axial velocity and radial velocity. The removal of surface materials partly depends on a considerable shearing action of water jets issuing from the helix nozzle. Furthermore, the spiral line added to the helix nozzle's inner surface had no distinctly opposite influence on axial velocity.

Cleaning effectiveness was obvious only when the velocity value was above $150 \mathrm{~m} / \mathrm{s}$. The curve denoted $R 90$ nozzle shows an effective cleaning range of about $0.078-0.12 \mathrm{~m}$ (the length of nozzle in our case was $0.078 \mathrm{~m}$ ), while the effective 


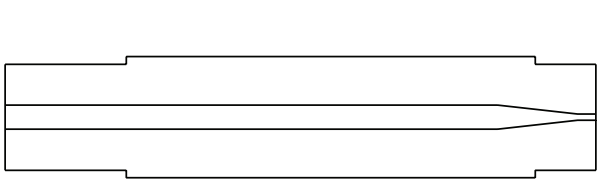

(a)

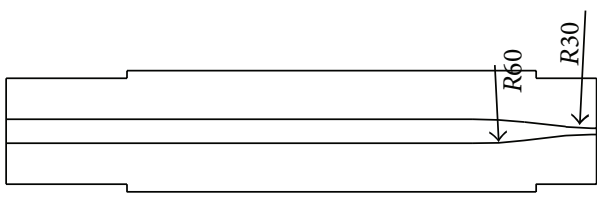

(c)

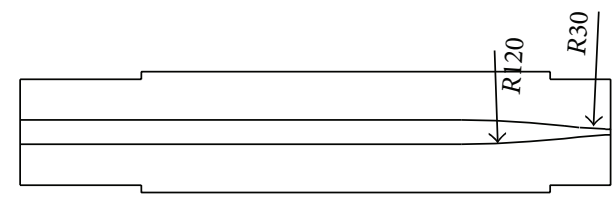

(e)

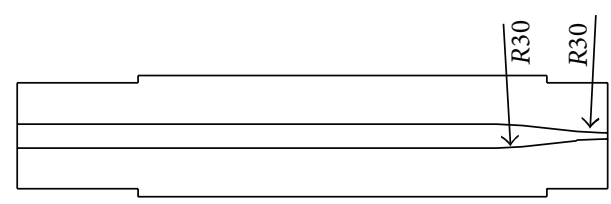

(b)

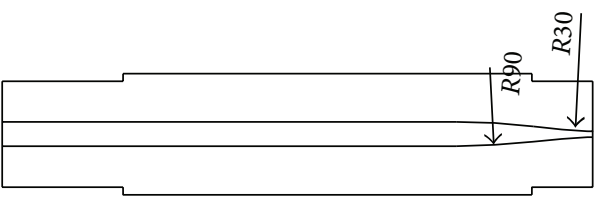

(d)

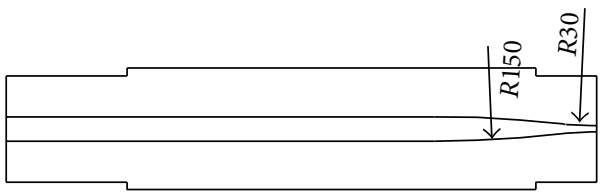

(f)

FIGURE 8: Preliminary structure optimization of CC nozzle.

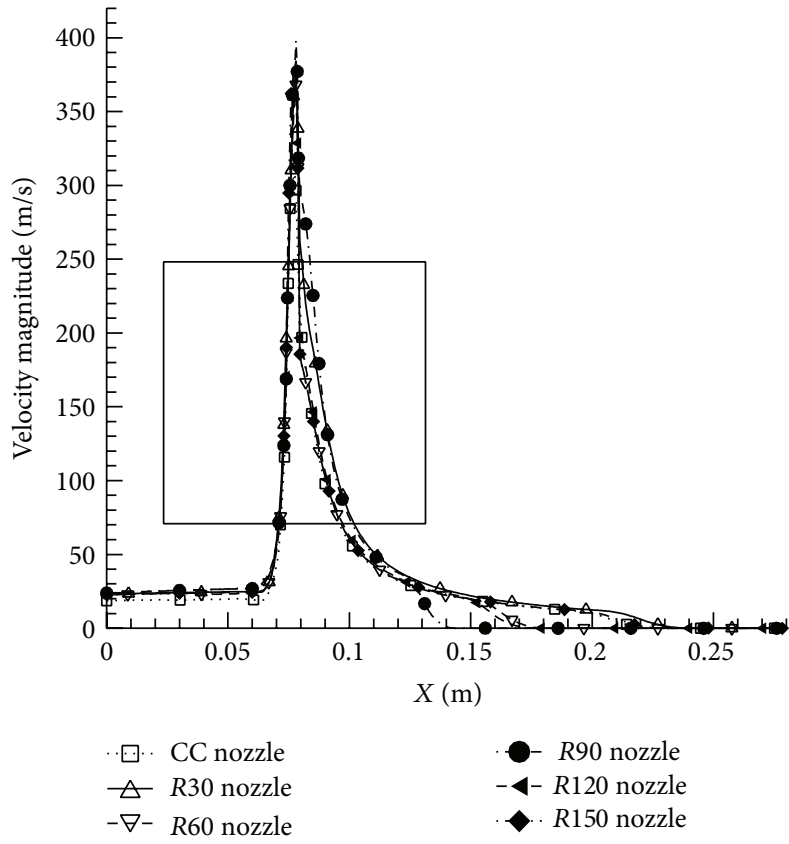

(a)

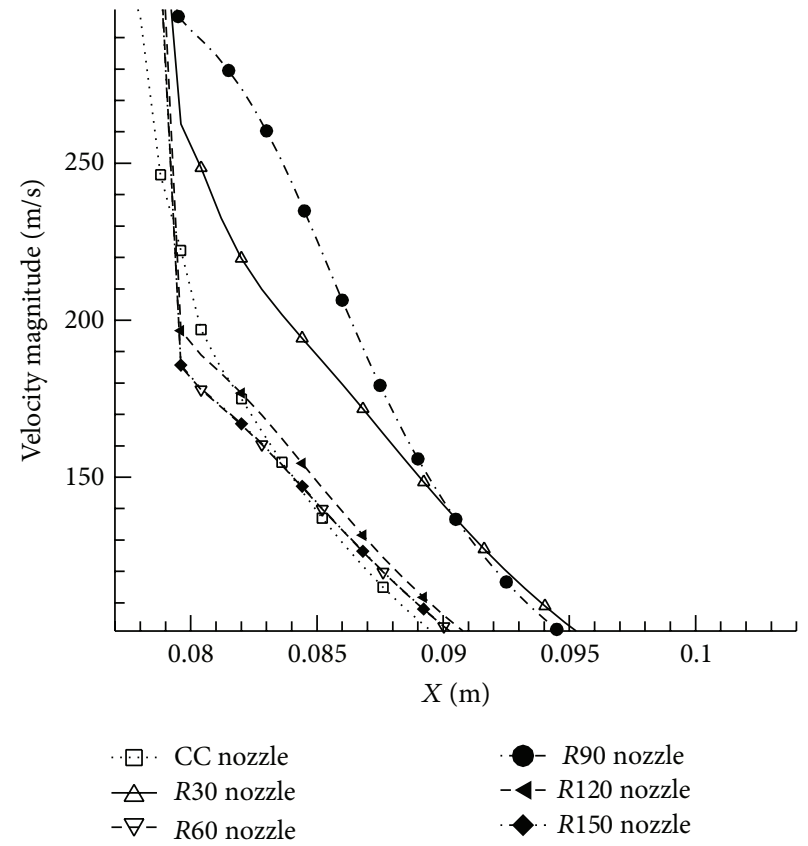

(b)

FIGURE 9: Jet velocity magnitude of preliminarily optimized CC nozzles compared with CC nozzle.

cleaning range of the helix nozzle was about $0.078-0.143 \mathrm{~m}$. In a specific limit, a wider effective cleaning range produces a broader jet width, resulting in more effective cleaning.

\section{Conclusion}

In this paper, an effective optimization of nozzle configuration for high pressure water jetting has been performed. The qualitatively demonstrable superiority and reliability of the designed object, which shows better manifestation of dynamic pressure, cohesion, and low local resistance loss, are also provided. As previous studies show, flow around the variable-area channel can dramatically change, where particles collide and produce a whirlpool, causing local resistance loss. To remedy this, we construct a highly effective, pliable structure in the nozzle's inner surface which creates an optimized structure, based on the principle of a helical accelerator. The governing equations of turbulence proposed by Tu et al. (2007) [7] were used to simulate our models. At the first step of optimization, nozzle $R 90$ (Figure $8(\mathrm{~d})$ ) 


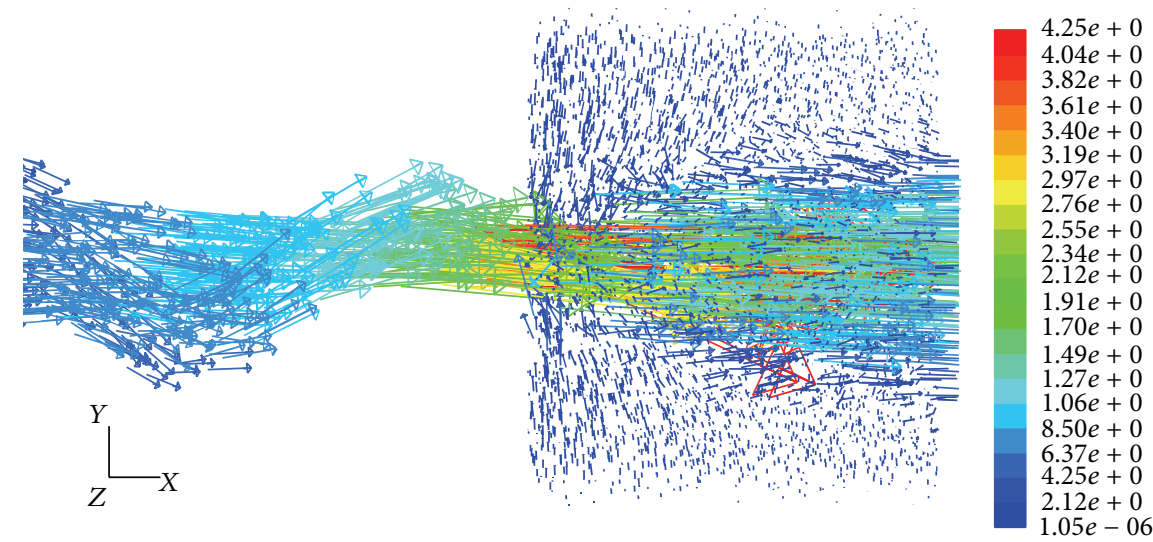

Velocity vectors colored by velocity magnitude $(\mathrm{m} / \mathrm{s})$

Jan 29, 2015

FLUENT 6.3 (3d, pbns, ske)

FIgURE 10: Velocity vectors of helix nozzle.

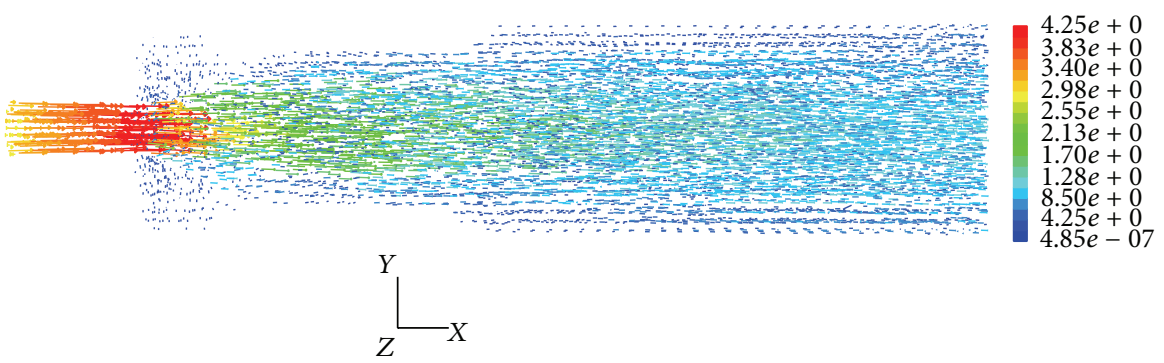

Velocity vectors colored by velocity magnitude $(\mathrm{m} / \mathrm{s})$

Jan 29, 2015

FLUENT 6.3 (3d, pbns, ske)

(a) Velocity vector of $R 90$ nozzle
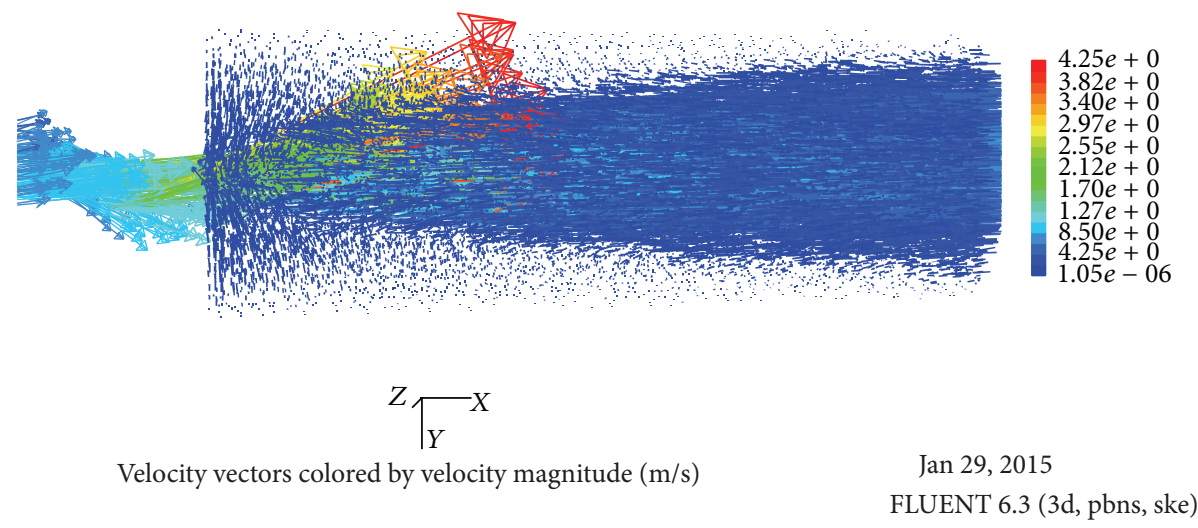

(b) Velocity vector of helix nozzle

FIGURE 11: Comparison of velocity vectors of $R 90$ nozzle and helix nozzle.

was chosen for subsequent experimentation. The relevant advantages and feasibility of adding a helix to the nozzle at the outlet were confirmed by applying rifle theory.

In future study, it is necessary to comprehensively analyze the quantity and basic parameters of a spiral line and its corresponding jetting divergency phenomenon in air, considering factors of cavitation and gravity. Further research on jetting performance by surface stress analysis of different cleaning objects will be discussed in the future.

\section{Conflict of Interests}

The authors declare that there is no conflict of interests regarding the publication of this paper. 


\section{Acknowledgments}

The authors acknowledge the financial support from Dalian Modern Auxiliary Machines Development and Manufacturing Co. and the purely technical help provided by Zhiyuan Jin from Dalian Polytechnic University and Pengtao Yi from Dalian Jiaotong University.

\section{References}

[1] W. H. Nurick, "Orifice cavitation and its effect on spray mixing," Journal of Fluids Engineering, vol. 98, no. 4, pp. 681-687, 1976.

[2] M. Hashish and M. P. du Plessis, "Prediction equations relating high velocity jet cutting performance to stand off distance and multipasses," Journal of Manufacturing Science and Engineering, vol. 101, no. 3, pp. 311-318, 1979.

[3] H. Soyama, Y. Yanauchi, K. Sato, T. Ikohagi, R. Oba, and R. Oshima, "High-speed observation of ultrahigh-speed submerged water jets," Experimental Thermal and Fluid Science, vol. 12, no. 4, pp. 411-416, 1996.

[4] D. Gao and J. Chen, "Experimental research on waterjet street surface cleaning," Proceedings of the Institution of Civil Engineers: Municipal Engineer, vol. 159, no. 2, pp. 71-75, 2006.

[5] K. Yanaida and A. Ohashi, "Flow characteristics of water jet in air," in Proceeding of the 5th International Symposium on Jet Cutting Technology, Paper A3, pp. 33-44, 1980.

[6] M. C. Leu, P. Meng, E. S. Geskin, and L. Tismeneskiy, "Mathematical modeling and experimental verification of stationary waterjet cleaning process," Journal of Manufacturing Science and Engineering, Transactions of the ASME, vol. 120, no. 3, pp. 571579, 1998.

[7] J. Tu, G. H. Yeoh, and C. Liu, Computational Fluid Dynamics: A Practical Approach, Elsevier Science, 2007.

[8] A. Guha, R. M. Barron, and R. Balachandar, "An experimental and numerical study of water jet cleaning process," Journal of Materials Processing Technology, vol. 211, no. 4, pp. 610-618, 2011.

[9] A. Guha, R. M. Barron, and R. Balachandar, "Numerical simulation of high-speed turbulent water jets in air," Journal of Hydraulic Research, vol. 48, no. 1, pp. 119-124, 2010.

[10] B. E. Launder and D. B. Spalding, "The numerical computation of turbulent flows," Computer Methods in Applied Mechanics and Engineering, vol. 3, no. 2, pp. 269-289, 1974. 

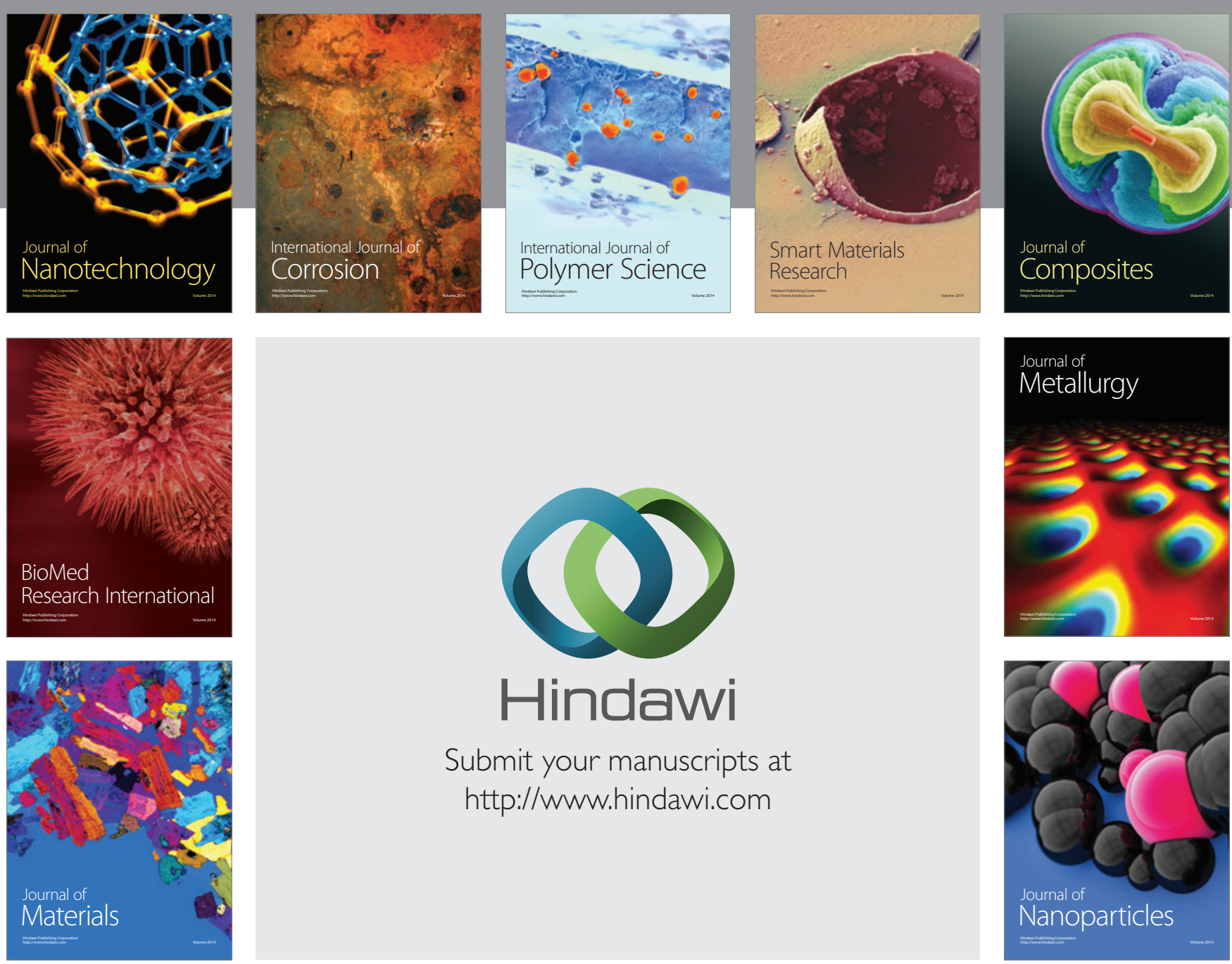

Submit your manuscripts at http://www.hindawi.com
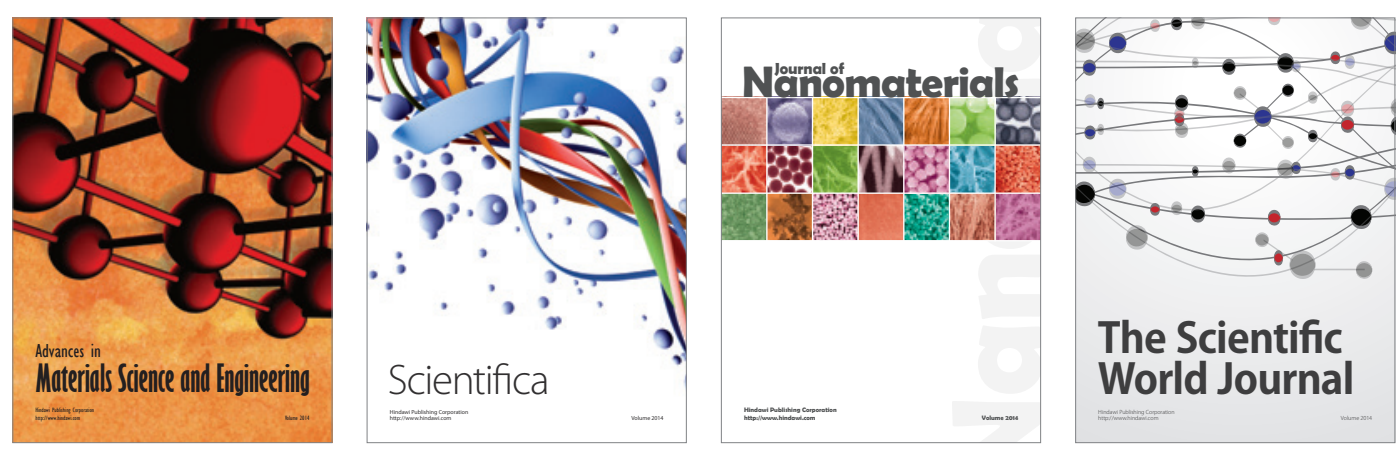

\section{The Scientific World Journal}
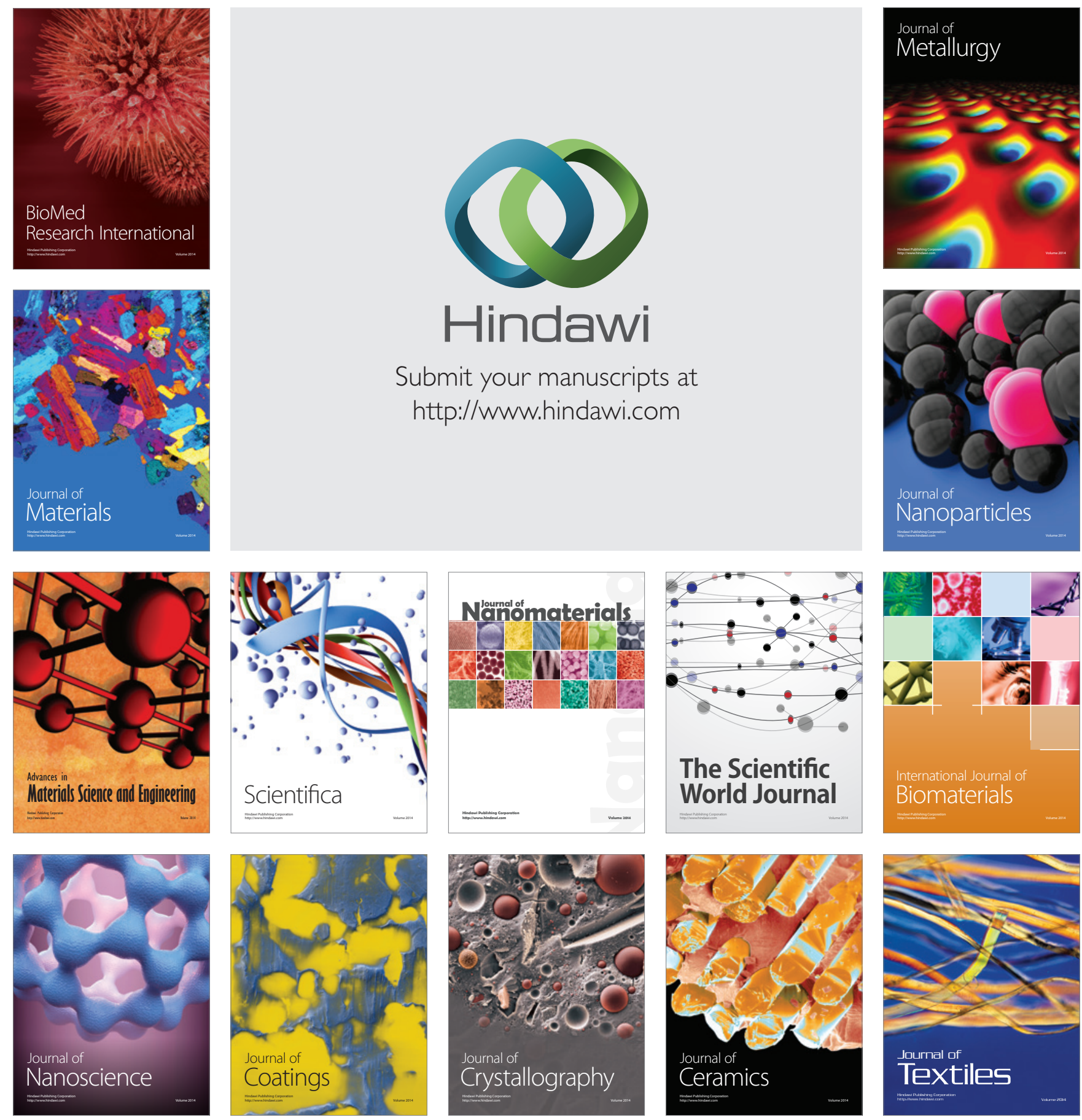